\title{
Silica-gelatin hybrids for tissue regeneration: inter-relationships between the process variables
}

\author{
Oliver Mahony $\cdot$ Sheng Yue $\cdot$ Claudia Turdean-Ionescu • \\ John V. Hanna • Mark E. Smith • Peter D. Lee • \\ Julian R. Jones
}

Received: 30 July 2013/Accepted: 11 November 2013/Published online: 27 November 2013

(C) The Author(s) 2013. This article is published with open access at Springerlink.com

\begin{abstract}
Owing to their diverse range of highly tailorable material properties, inorganic/organic hybrids have the potential to meet the needs of biodegradable porous scaffolds across a range of tissue engineering applications. One such hybrid platform, the silica-gelatin sol-gel system, was examined and developed in this study. These hybrid scaffolds exhibit covalently linked interpenetrating networks of organic and inorganic components, which allows for independent control over their mechanical and degradation properties. A combination of the sol-gel foaming process and freeze drying was used to create an interconnected pore network. The synthesis and processing of the scaffolds has many variables that affect their structure and properties. The focus of this study was to develop a matrix tool that shows the inter-relationship between process variables by correlating the key hybrid material properties with the synthesis parameters that govern them. This was achieved by investigating the effect of the organic (gelatin) molecular weight and collating previously reported data.
\end{abstract}

O. Mahony · J. R. Jones $(\bowtie)$

Department of Materials, Imperial College London, London SW7 2AZ, UK

e-mail: julian.r.jones@imperial.ac.uk

S. Yue $\cdot$ P. D. Lee

Manchester X-ray Imaging Facility, School of Materials, University of Manchester, Oxford Road,

Manchester M13 9PL, UK

C. Turdean-Ionescu $\cdot$ J. V. Hanna $\cdot$ M. E. Smith

Department of Physics, University of Warwick,

Coventry CV4 7AL, UK

M. E. Smith

Vice-Chancellor's Office, Lancaster University,

Lancaster LA1 4YW, UK
Control of molecular weight of the polymer is as an avenue that allows the modification of hybrid material properties without changing the surface chemistry of the material, which is a factor that governs the cell and tissue interaction with the scaffold. This presents a significant step forward in understanding the complete potential of the silica-gelatin hybrid system as a medical device.

Keywords Gelatin - GPTMS · Hybrid - Scaffold · Sol-gel $\cdot$ Foaming $\cdot$ Covalent coupling

\section{Introduction}

Advanced tissue regeneration platforms have been developed based on porous hybrid materials containing silica and gelatin. A hybrid material consists of interpenetrating networks of organic and inorganic components (Fig. 1) [1-8]. The properties of hybrids are customisable and can be tailored precisely to the needs of their intended application. For instance, by varying the inorganic and organic content, these hybrid materials can be designed as soft and flexible or hard and stiff. They can even be modified in order to exhibit slow or fast dissolution rates through the degree of covalent coupling [9]. Hybrids present significant advantages over conventional composites, in which it is often difficult to control properties such as dissolution rate and surface chemistry characteristics due to differential dissolution of the components and masking of the bioactive component [8]. The diverse range of hybrid material properties are attributed, largely, to their unique chemical structure due to the nanoscale interaction between the components and covalent links between the inorganic and organic phases (Fig. 1) [10]. The covalent links are facilitated by reaction with organosilanes such as 3-glycidoxypropyltrimethoxysilane (GPTMS) [11-14], 


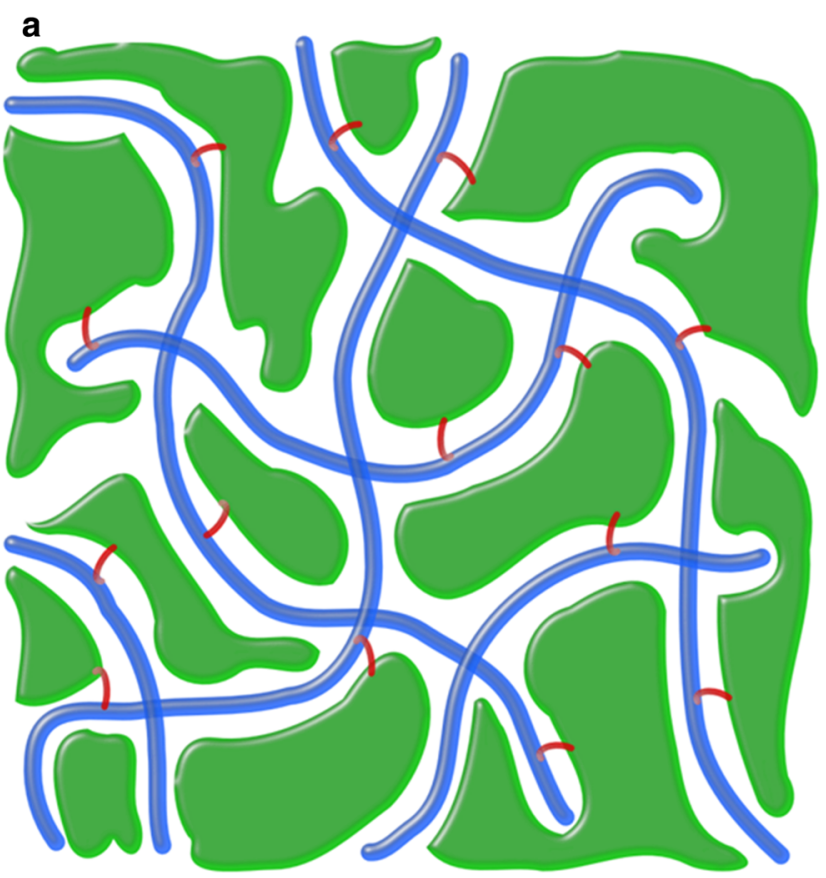

\section{b}

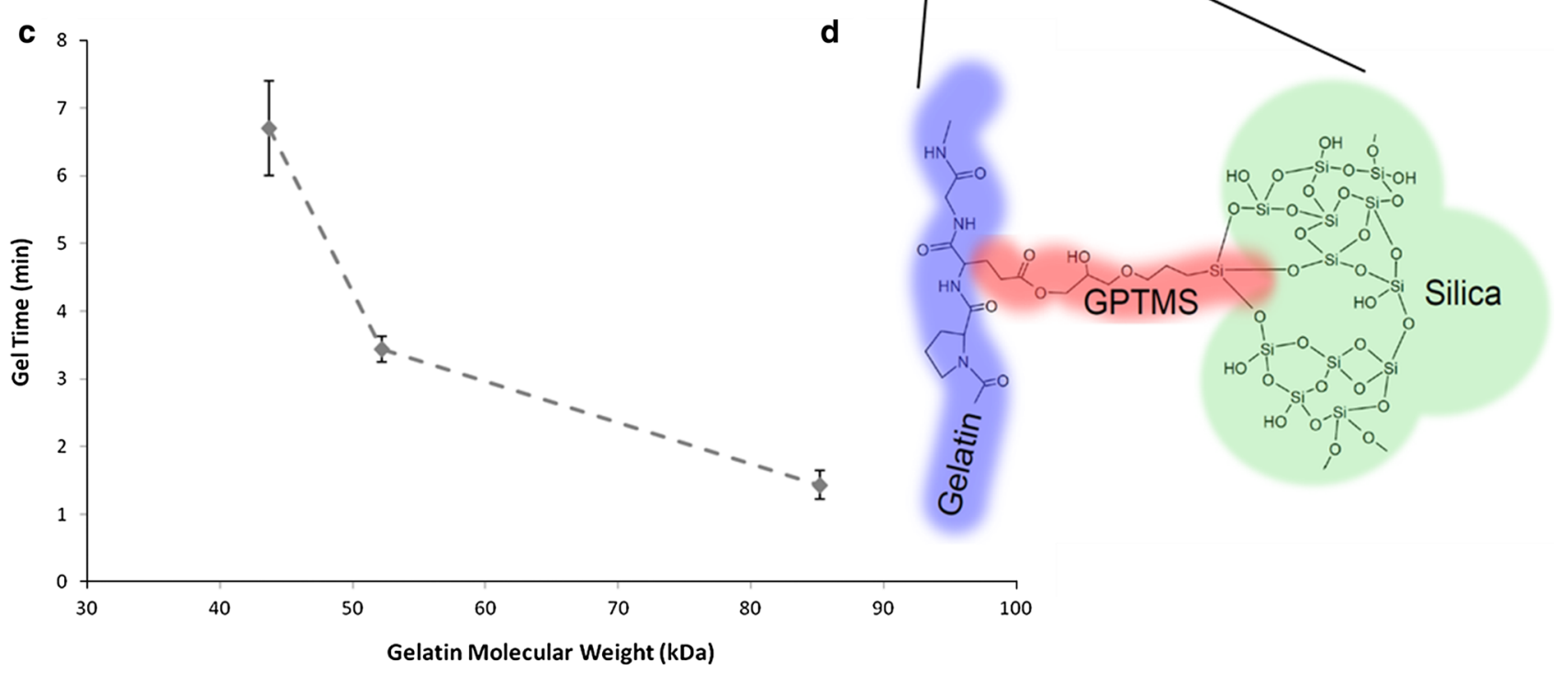

Fig. 1 Hybrid condensation reaction kinetics as a function of gelatin molecular weight. Schematics representing the linking of inorganic and organic networks in the hybrid on a molecular scale for the high molecular weight gelatin (a) and the low molecular weight gelatin (b). Blue indicates gelatin chains, green is the silica phase and red is

the GPTMS bridging molecule. The effect of gelatin molecular weight as a function of hybrid gelation time with a HF sol concentration of $0.1 \%(\mathrm{v} / \mathrm{v})(\mathbf{c})$. The chemical structure that represents the link between the organic and inorganic phases (d) (Color figure online)

3-aminopropyltriethoxysilane (APTES) [15-17] and 3-isocyanatopropyltriethoxysilane (ICPTES) $[6,18,19]$. In this work coupling was provided by GPTMS, which bonds to the nucleophilic functional groups of gelatin on one end [20,21], whilst hydrolysing and condensing on the other end to react with the silica network as the network forms (Fig. 1d) [9]. This bridging molecule is fundamental in achieving good control over the nanoscale phase interaction, the mechanical properties and the dissolution characteristics as shown through previous work [9]. It is due to this bridging molecule that the

material is a true hybrid, behaving as one material phase, while exploiting the benefits of each of the individual phases. Gelatin was used in this system as it is a hydrolysed form of collagen which is recognisable and adhered to by many cell types [22]. And silica is well documented in biocompatible applications [23-25]. Consequently silica-gelatin hybrids also display excellent cell compatibility in vitro with mesenchymal stem cells [9].

In this study the full potential of the material properties of silica-gelatin hybrids are explored by defining the 
interelationship between the key variables and the hybrid material properties. This is defined by producing a matrix of material properties that can be used in future design of hybrid materials. To produce this matrix a number of hybrid processing parameters were investigated. The effect of gelatin molecular weight on hybrids was investigated with a view to altering mechanical properties while minimising the changes in material surface chemistry, in order to maintain the favourable cell interactions exhibited by silica-gelatin hybrids. This study considered hybrids with a $60 \mathrm{wt} \%$ organic content and a C-factor (the molar ratio of GPTMS/gelatin for the highest molecular weight gelatin used) of 750 .

\section{Experimental section}

All reagents were supplied by Sigma Aldrich, UK. Hybrids were made with gelatin (Type A, porcine) of 3 different molecular weights. The labels B100, B175 and B300 were given corresponding to the bloom number of the respective gelatin samples (a number that quantifies the molecular weight based on viscosity measurements). Gel permeation chromatography (GPC) was carried out to determine the molecular weight of gelatin. Samples were dissolved in $0.2 \mathrm{M} \mathrm{NaNO}_{3}, 0.01 \mathrm{M} \mathrm{NaH}_{2} \mathrm{PO}_{4}(\mathrm{pH} 7.0)$ with a polymer concentration of $2 \mathrm{mg} \mathrm{ml}^{-1}$. Analysis was carried out on a Viscotek Triple Detector Array TDA301 equipped with PL aquagel guard plus two mixed-OH $(30 \mathrm{~cm} 8 \mu \mathrm{m})$ columns and a refractive index detector (with differential pressure and light scattering). The system was calibrated using pullulan polysaccharides. The sample temperature was $35{ }^{\circ} \mathrm{C}$ and the flow rate was $1 \mathrm{ml} / \mathrm{min}$. Data was analysed with Malvern/Viscotek OmniSec software.

Hybrids were synthesised by a method described in full elsewhere [9]. Initially, gelatin $\left(50 \mathrm{mg} \mathrm{ml}^{-1}\right)$ was functionalised by reaction with GPTMS, which grafted silane groups onto the gelatin chain, in dilute hydrochloric acid $(\mathrm{HCl})$ at $\mathrm{pH} 5$ for $14 \mathrm{~h}$ at $40{ }^{\circ} \mathrm{C}$. The mass ratio of GPTMS/ gelatin was kept constant at 2.03, which was equivalent to a C-factor (molar ratio of GPTMS/gelatin) of 750 for the highest molecular weight gelatin-B300. Functionalised gelatin was then used as a precursor in the sol-gel process, which was carried out as follows: tetraethylorthosilicate (TEOS) was added to $0.25 \mathrm{M} \mathrm{HCl}$ solution with a water/ TEOS molar ratio of 4. TEOS was left to hydrolyse for $1 \mathrm{~h}$ before adding an appropriate amount of functionalised gelatin to the sol to give a final hybrid with organic content of $60 \mathrm{wt} \%$. The sol was allowed to mix for a further hour at $40{ }^{\circ} \mathrm{C}$ where the network forming condensation reaction between hydrolysed TEOS and functionalised gelatin began. Foaming of the sol then took place by adding $1 \mathrm{ml}$ of gelling agent hydrofluoric (HF) acid (5\% (v/v)) to a
$50 \mathrm{ml}$ sol aliquot together with a surfactant-Triton X-100. The sol was vigorously mixed until the point of gelation when the resultant foam was poured into sealed $60 \mathrm{ml}$ vials. Foaming was carried out with varied Triton $\mathrm{X}-100$ concentrations in the sol $(0.2,0.5,1$ and $2 \%(\mathrm{v} / \mathrm{v}))$. The gelation time and total foam volume was noted. Hybrids were aged at room temperature for 4 days and then dried by freeze drying (Thermo Scientific Heto PowerDry LL 1500 ) at a pressure of 0.13 mbar. Some samples were also aged at $40{ }^{\circ} \mathrm{C}$ and $60{ }^{\circ} \mathrm{C}$ to investigate the effects of aging on their atomic structure using ${ }^{29} \mathrm{Si}$ magic angle spinning nuclear magnetic resonance (MAS NMR) spectroscopy. Measurements were carried out on a Varian InfinityPlus $300 \mathrm{MHz}$ spectrometer operating at $59.62 \mathrm{MHz}$, using MAS of $4 \mathrm{kHz}$ with a $20 \mathrm{~s}$ recycle delay and pulses with a tip angle of $<30^{\circ}$. Chemical shifts were referenced externally to TMS at $0 \mathrm{ppm}$.

Cylindrical scaffolds were punched from bulk foams and cut with a diameter/thickness ratio of 1:1 and underwent compressive testing (Instron 5866) with a $500 \mathrm{~N}$ load cell and a compression extension speed of $0.5 \mathrm{~mm} / \mathrm{min}$ $(\mathrm{n}=10)$.

Scanning electron microscopy (SEM) was carried out on gold sputter coated samples with a 5610 LV SEM (JEOL, USA). An accelerating voltage of $15 \mathrm{kV}$ and spot size of $35 \mu \mathrm{m}$ were used. Pore interconnect size quantification was carried out on Image J software $(n>200)$.

The scaffold skeletal density, $\rho_{s}$, was determined by helium pycnometry (Quantachrome Pycnometer, UK) and the scaffold bulk density, $\rho$, was found through scaffold mass and geometry measurements.

Cylindrical scaffolds, $8 \mathrm{~mm}$ in diameter and $5 \mathrm{~mm}$ high, was scanned using a X-ray microtomography (micro-CT) unit (Phoenix X-ray Systems and Services $\mathrm{GmbH}$, Wunstorf, Germany) with acceleration voltage of $55 \mathrm{kV}$ and filament current of $180 \mu \mathrm{A}$, and with a voxel size of $10 \mu \mathrm{m}$. After imaging, a $3 \times 3 \times 3$ median filter was applied to the micro-CT volume $(1,015 \times 1,015 \times 512$ voxels in size) for noise reduction. Then the volume was binarised by applying a threshold with equidistant to the background peak and scaffold peak in the histogram respectively. Pore size distributions were calculated using a technique that was developed for these materials, Due to the high porosity and the morphology of the pore network in the scaffold, the pore size distribution was described using an accessible volume algorithm [26], which is analogous to mercury porosimetry. The alternative was to measure individual pores, but this requires segmentation of the pores into unique entities [27], which is almost impossible in this case due to the open nature of the network. The accessible volume algorithm measured the percentage of volume in the scaffold that can be accessed (termed accessible volume) by a virtual sphere from 
outside of the scaffold. By gradually increasing the size of the virtual sphere, the variation of the accessible volume in the open porous scaffold was recorded and the incremental pore volume distribution was calculated.

Like any CT based quantification, the accuracy of the results at the fine scale is limited by the voxel size. Here, smaller pores with equivalent sphere diameter less than $50 \mu \mathrm{m}$ may be underestimated in size, but for the majority of the pore space, the voxel size was with adequate resolution to describe the pore network.

Hybrid scaffolds $(100 \mathrm{mg}$ ) were immersed in $100 \mathrm{ml}$ simulated body fluid (SBF) [28] and left on an orbital shaker at $120 \mathrm{rpm}$ and $37^{\circ} \mathrm{C}$. At each time point throughout the 30 day study $1 \mathrm{ml}$ sample was removed and replenished with SBF. $1 \mathrm{ml}$ samples from each time point $(\mathrm{n}=3)$ were analysed for gelatin concentration (Pierce Micro BCA protein assay, with gelatin solution standard $(\mathrm{n}=3))$ and silicon, calcium and phosphorus concentration using inductively coupled plasma spectroscopy (ICP) (PerkinElmer Optima 2000DV, flow rate of $1.5 \mathrm{ml} / \mathrm{min}$ and plasma power of $1,300 \mathrm{~W}(\mathrm{n}=3))$.

Powder X-ray diffraction (XRD) was performed on samples immersed for 20 days in SBF. Samples were rinsed and ground before being examined on a PANalytical X'Pert PRO with a $2 \theta$ range of $5^{\circ}-70^{\circ}$, a step size of $0.04^{\circ}$ and $X$-ray collection time of $0.25 \mathrm{~s}$ at each step. Data was analysed with HighScore Plus software (PANalytical, Holland).

\subsection{Theory}

Cellular solid theory was employed to determine the skeletal strength and stiffness of hybrid scaffolds with varying porosity [29]. The model was validated for silica-gelatin hybrids in previous work [9]. This method effectively normalised against the effect of the varied porosity amongst the scaffolds tested. The skeletal stiffness, $E_{s}$ was found from

$E=E_{s}\left(\frac{\rho}{\rho_{s}}\right)^{2}$

where $E$ is the measured scaffold stiffness and the term $\rho / \rho_{s}$ is the relative density, $\rho_{r}$. Relative density is an equivalent measure to porosity, $\Phi\left(\Phi=1-\rho_{r}\right)$ The scaffold failure mode is through plastic collapse and the plastic collapse strength, $\sigma_{p l}$, which was measured experimentally, is expressed as

$\sigma_{p l}=0.3 \sigma_{y}\left(\frac{\rho}{\rho_{s}}\right)^{\frac{3}{2}}$

where, $\sigma_{y}$ is the skeletal strength of the hybrid.

Relative density was calculated from bulk density, $\rho$, and skeletal density, $\rho_{s},\left(\rho_{r}=\rho / \rho_{s}\right)$. This was used to estimate pore interconnect diameter, $d$ (in micrometers),
Table 1 Molecular weight and polydispersity of gelatin used for hybrid synthesis determined from conventional GPC and triple detection methods

\begin{tabular}{llll}
\hline Gelatin sample & $\mathrm{M}_{\mathrm{w}}(\mathrm{kDa})$ & $\mathrm{M}_{\mathrm{n}}(\mathrm{kDa})$ & $\mathrm{M}_{\mathrm{w}} / \mathrm{M}_{\mathrm{n}}$ \\
\hline B100 & 43 & 3.1 & 14 \\
B175 & 52 & 3.5 & 15 \\
B300 & 85 & 5.7 & 15 \\
\hline
\end{tabular}

Sample names indicate the bloom number of the gelatin sample provided by the manufacturer

based on a previous model developed by geometric considerations [9].

$\rho_{r}^{\frac{1}{3}}=1.03 \times 10^{2} d+1$

To determine the network connectivity with quantitative NMR results the from the calculated percentage values of $\mathrm{Q}$ and $\mathrm{T}$ species, the following equation was used:

connectivity $=\frac{1 T^{1}+2 T^{2}+3 T^{3}}{3}+\frac{2 Q^{2}+3 Q^{3}+4 Q^{4}}{4}$

where $Q^{n}$ represents a silicon atom bound to $n$ bridging oxygens in a silicate network and $T^{n}$ represents a silicon atom bound to one carbon plus $n$ bridging oxygens of a silicate network [30].

\section{Results and discussion}

The batches of gelatin used for the hybrid synthesis were characterised with GPC to assess their weight average molecular weight $\left(\mathrm{M}_{\mathrm{w}}\right)$, number average molecular weight $\left(\mathrm{M}_{\mathrm{n}}\right)$ and polydispersity $\left(\mathrm{M}_{\mathrm{w}} / \mathrm{M}_{\mathrm{n}}\right)$. Conventional GPC data gave $M_{w}$ and $M_{n}$ relative to a pullulan polysaccharide standard. These were not absolute values, but the relative comparisons between the samples were valid. In order to determine the true molecular weight triple detection GPC was carried out on the largest gelatin polymer (B300), which was found to have a $\mathrm{M}_{\mathrm{w}}$ of $85 \mathrm{kDa}$. The triple detection technique is generally considered to give larger sources of error relating to polymer concentration, but the reported value agreed with the estimated value claimed by the supplier of the polymer. Based on the relative differences between polymers discovered through conventional GPC the molecular weight and polydispersity were estimated (Table 1).

\subsection{Mechanism of gelation and generation of porosity}

As silica-gelatin hybrids are synthesised via a sol-gel foaming route, the gelation mechanism is of significant 
importance. To make scaffolds, the sol of hydrolysed TEOS and functionalised polymer is foamed under vigorous agitation and the bubbles are stabilised with the aid of a surfactant $[31,32]$. Gelation of the sol must coincide with the formation of a stable foam structure [33, 34]. On gelation, the foam is stable and surfactant films rupture, connecting the pores. In sol-gel glass foaming [31], the silica network gels but when gelatin is present, the gelatin can also gel. However gelatin gelation is thermally reversible, so silica network formation is necessary to produce a useful hybrid. Surfactant concentration and gelation time are critical variables for the foaming process [33]. In the current study, when the molecular weight of gelatin decreased, the gelation time increased (Fig. 1c). Sols containing the lowest molecular weight gelatin (43 kDa) gelled after 6.5 min which was $\sim 5$ min longer than sols with the $85 \mathrm{kDa}$ polymer. The change observed in the condensation reaction kinetics of the 3 hybrid sols is best explained by reference to the schematic in Fig. 1a, b. In order for the hybrid sol to gel, a continuous chemical network forms between the inorganic and organic chains via the GPTMS bridging molecule. The sol is created by hydrolysing TEOS. The silica network begins to form through polycondensation of $\mathrm{Si}-\mathrm{OH}$ groups, forming $-\mathrm{Si}-$ $\mathrm{O}-\mathrm{Si}-$ bonds. Silica nanoparticles begin to coalesce and form a network through further condensation [35]. Prior to addition of the gelatin to the sol of hydrolysed TEOS, the gelatin was functionalised by reaction with GPTMS [9], through reaction of nucleophilic groups with the epoxy ring. To complete the network formation process, the silane groups of GPTMS must hydrolyse and undergo condensation polymerisation with the silica network (Fig. 1d). This process was catalysed by the addition of HF. The sol gelled partly due to the formation of a rigid silica network, but also due to the effect of immobilising the gelatin chains by chemically linking them to the silica network (also catalysed by HF due to the condensation of $\mathrm{Si}-\mathrm{OH}$ functional groups with the silica network). When the sol contained gelatin chains of lower molecular weight there were more chains to immobilise via the bridging action of GPTMS to the inorganic network (Fig. 1b, c). Therefore to form a gel more GPTMS molecules had to react in order to immobilise all of the gelatin chains, increasing the gelation time. However, the reaction rate increased when HF concentration was increased. It is important to consider the gelation time when foaming as this is fundamental to stable foam formation as the increase in viscosity that occurs during gelation helps to stabilise the foam [33]. An ideal gelation time is between 4-7 min to allow the formation of a suitable foam structure. HF concentration can be adjusted to meet this requirement.

The technique of foaming, ageing and drying enabled the production of highly porous scaffolds with porosities of up to $97.7 \%$. Figure 2a shows a micro-CT image of a hybrid of $90 \%$ porosity, made with $60 \mathrm{wt} \%$ gelatin. The very open and interconnected structure (Fig. 2a) provides a suitable environment to allow for complete penetration of cells throughout the scaffold. The porosity of these highly porous scaffolds could not be evaluated by the standard method of mercury intrusion porosimetry, as the pores were too large. However, quantitative analysis of micro-CT images by the accessible volume method gave macropore interconnect size distribution (Fig. 2c). Whilst there was a reasonably broad interconnect size distribution found between 300 and $600 \mu \mathrm{m}$, there was one clear mode at $360 \mu \mathrm{m}$. This is a large pore interconnect size for a tissue scaffold, particularly when an interconnect diameter of $100 \mu \mathrm{m}$ is considered by many to be sufficient [36]. Therefore, there is large scope for varying the scaffold porosity, by modifying foaming and ageing parameters, in order to tailour mechanical properties without sacrificing cell penetration capabilities.

Quantitative micro-CT data was verified by manual pore interconnect measurement from SEM (Fig. 2b). The pore interconnect size distribution results for this technique (Fig. 2c) agreed with the $\mu \mathrm{CT}$ results, giving a modal interconnect diameter close to $360 \mu \mathrm{m}$. This confirmed the highly porous nature of the hybrid scaffolds and validated the accessible volume algorithm used with this quantitative $\mu \mathrm{CT}$ technique.

\subsection{Influence of gelatin molecular weight}

The hybrid mechanical properties were evaluated by compressive testing. Previous studies showed hybrid scaffolds behaved according to a cellular solid model [9]. Therefore, in order to normalise against the effect of varied porosity across samples a cellular solid model [29] was employed (Sect. 2.1.) to calculate the skeletal strength and stiffness (Fig. 3a). The results show an increase in strength and stiffness as the molecular weight of gelatin increased. This was expected due to the increase in entanglement associated with the presence of longer gelatin chains. The increased entanglement led to a hybrid network that was more rigid and hence exhibited a stronger and stiffer bulk material.

The results presented in Fig. 3a show the strength/ stiffness for the skeletal material as if it were non-macroporous, but it is important to know the strength properties for porous scaffolds with the design requirements for an intended application. A typical design requirement for bone regenerating scaffolds is that they should have a pore interconnect diameter in excess of $100 \mu \mathrm{m}$ to allow for vascularisation and tissue ingrowth [36, 37]. If this value is used as a design parameter it can be input into a model (Sect. 2.1) developed for silica-gelatin hybrids to predict 

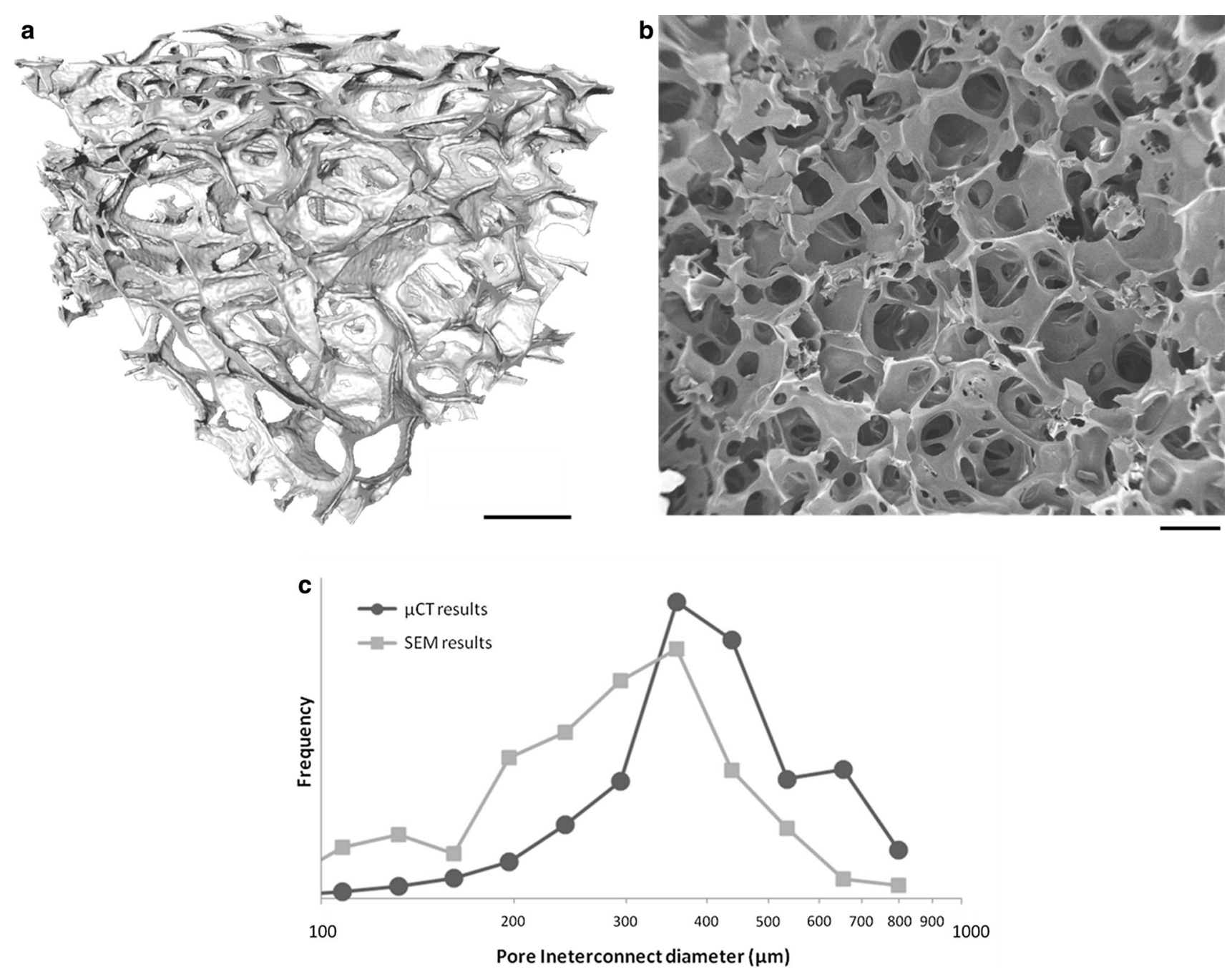

Fig. 2 Pore analysis of the $60 \mathrm{wt} \%$ gelatin hybrid scaffolds with $90 \%$ porosity. 3-dimensional $\mu \mathrm{CT}$ image of a hybrid scaffold (a) and SEM image of scaffold (b). Scale bars are $500 \mu \mathrm{m}$. Quantitative pore interconnect analysis with $\mu \mathrm{CT}$ and corresponding data from SEM analysis (c)

the corresponding percentage porosity [9]. For a $100 \mu \mathrm{m}$ pore interconnect diameter the porosity required was predicated to be $88 \%$. This porosity value can then be used with cellular solid theory to back calculate scaffold strength at this porosity based on the skeletal strength values already found (Fig. 3a). The predicted strengths for hybrid scaffolds with $88 \%$ porosity containing gelatin of varying molecular weight are shown in Fig. 3b. The hybrid strength varied between 1.2 and $2.0 \mathrm{MNm}^{-2}$ by controlling the molecular weight of gelatin used in the hybrids. Not only do these results show that the compressive strength is close to the 2-12 $\mathrm{MNm}^{-2}$ range of cancellous bone [38] for a highly porous scaffold, they also demonstrate that the molecular weight of gelatin can be used in the to control the mechanical properties. Furthermore, this method shows it is possible to alter the properties of the hybrids without altering the surface chemistry of the material, which means that hybrid scaffolds can be used as platforms to assess cell-substrate interactions with varying mechanical properties irrespective of the material chemistry.

Tweaking the porosity of the hybrid scaffolds provides control of their strength, so it is important to acquire good control of the porosity in the foaming process. During the foaming process a surfactant (Triton X-100) was added to lower the surface tension of the sol thereby stabilising the foam that is produced. The foam volume immediately prior to the point of gelation, from a starting point of $50 \mathrm{ml}$, was measured as a function of surfactant concentration (Fig. 4). As the concentration of the surfactant in the sol was increased the foam volume achieved in the foaming process also increased with a near linear relationship. When the surfactant was present in larger concentrations it created foams that were more stable and hence facilitated the formation of larger bubbles. Increasing foam volume produces an increase in pore size in the sol-gel foaming process [33]. 

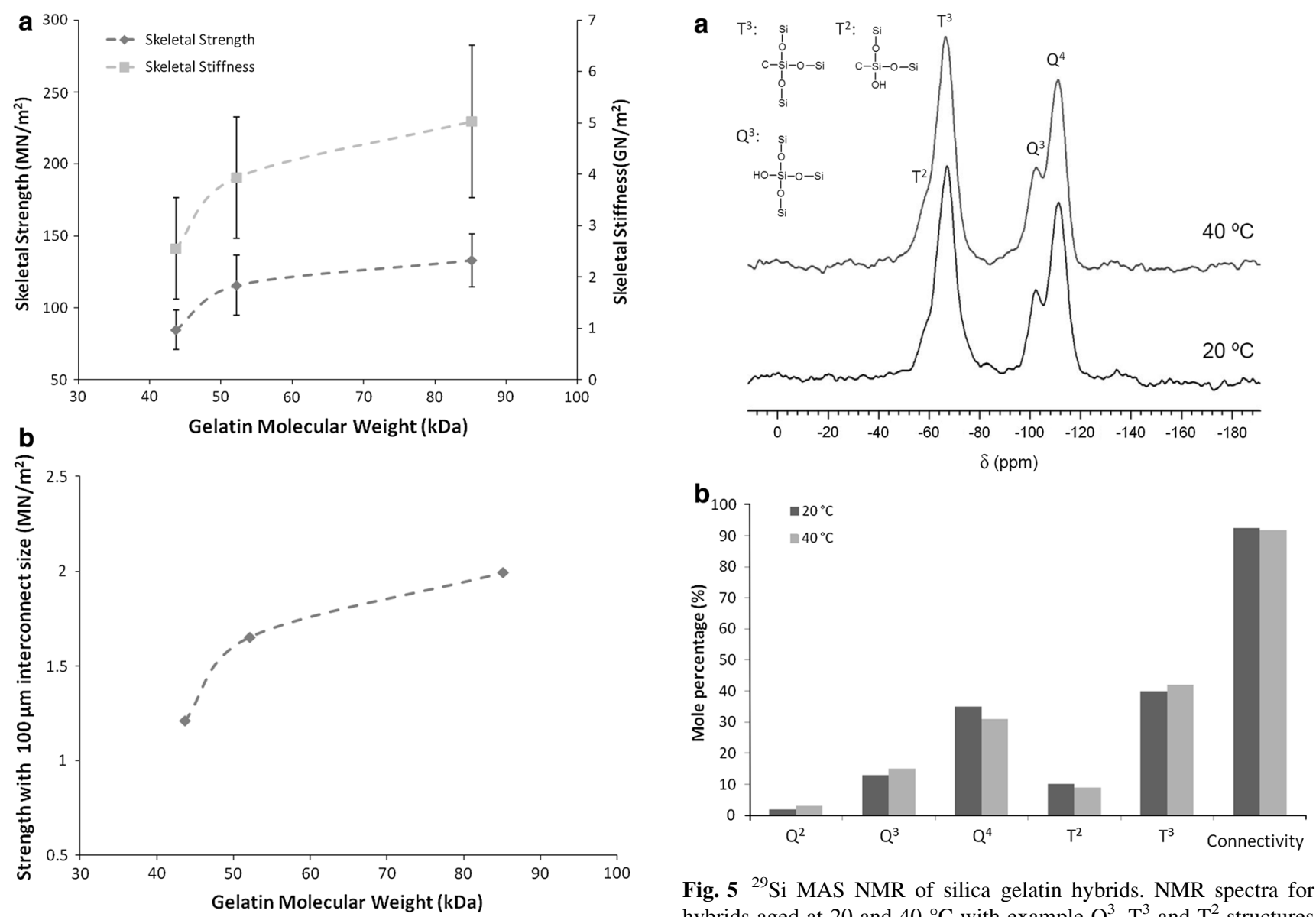

Fig. $5{ }^{29} \mathrm{Si}$ MAS NMR of silica gelatin hybrids. NMR spectra for hybrids aged at 20 and $40{ }^{\circ} \mathrm{C}$ with example $\mathrm{Q}^{3}, \mathrm{~T}^{3}$ and $\mathrm{T}^{2}$ structures (a) and respective quantification of the inorganic structure including the total network connectivity (b)

Fybrid scaffold mechanical properties. The skeletal strength and stiffness of $60 \mathrm{wt} \%$ gelatin scaffolds as a function of gelatin molecular weight (a). The predicted strength of a scaffolds with an interconnect diameter of $100 \mu \mathrm{m}$ containing gelatin at various molecular weights (b)

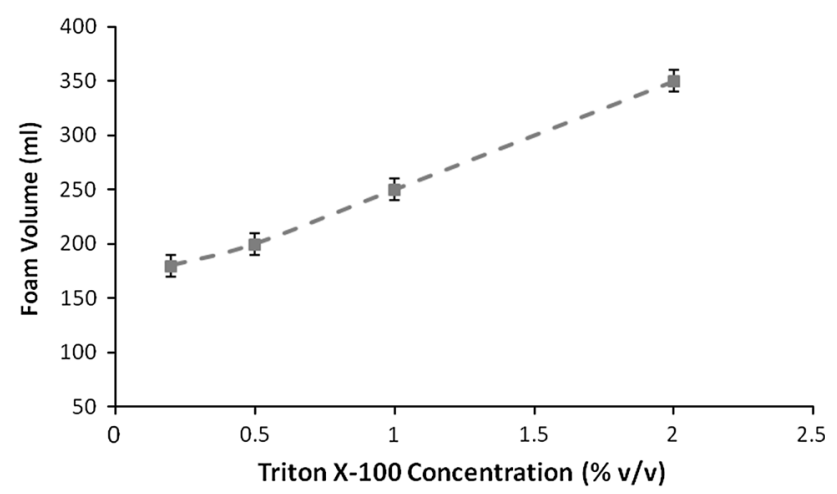

Fig. 4 The relationship of silica-gelatin hybrid foam volume and triton X-100 (surfactant) concentration in the sol-gel foaming process for hybrids containing gelatin across all molecular weights that were investigated

However, there is a second process that influences final porosity. This is the ageing and drying process. Ageing is a process that is carried out on gels in their wet state, in

sealed containers that prevent drying, usually at elevated temperatures to allow the silica network to further condense giving greater network connectivity [39]. Visibly the pores of the gel were observed to enlarge as the gel 'relaxed' during the ageing process. This was attributed to the gelatin phase exhibiting a viscous flow when wet. In the freeze drying process gels were observed to shrink due to the effect of water removal as well as the process of continued condensation. Ageing was carried out at 20,40 and $60{ }^{\circ} \mathrm{C}$. At $60{ }^{\circ} \mathrm{C}$ the gels deformed and so $60^{\circ} \mathrm{C}$ was considered too high. When ageing at $20{ }^{\circ} \mathrm{C}$ the gel volume was higher than at $40{ }^{\circ} \mathrm{C} .{ }^{29} \mathrm{Si}-\mathrm{NMR}$ revealed that hybrids aged in each manner had very similar inorganic structures (Fig. 5a). Quantification of this data (Fig. 5b) showed only very slight variation in the relative abundance of the respective $\mathrm{Q}$ and $\mathrm{T}$ species (see Fig. 5a for examples of $\mathrm{Q}$ and $\mathrm{T}$ species and Sect. 2.1 for further explanation). Summation of the $\mathrm{Q}$ and $\mathrm{T}$ species to give the degree of condensation (Sect. 2.1) revealed that the silica network connectivity was very similar. Therefore the observed variation of scaffold volume was attributed to a physical rearrangement of the gelatin chains in the organic phase 

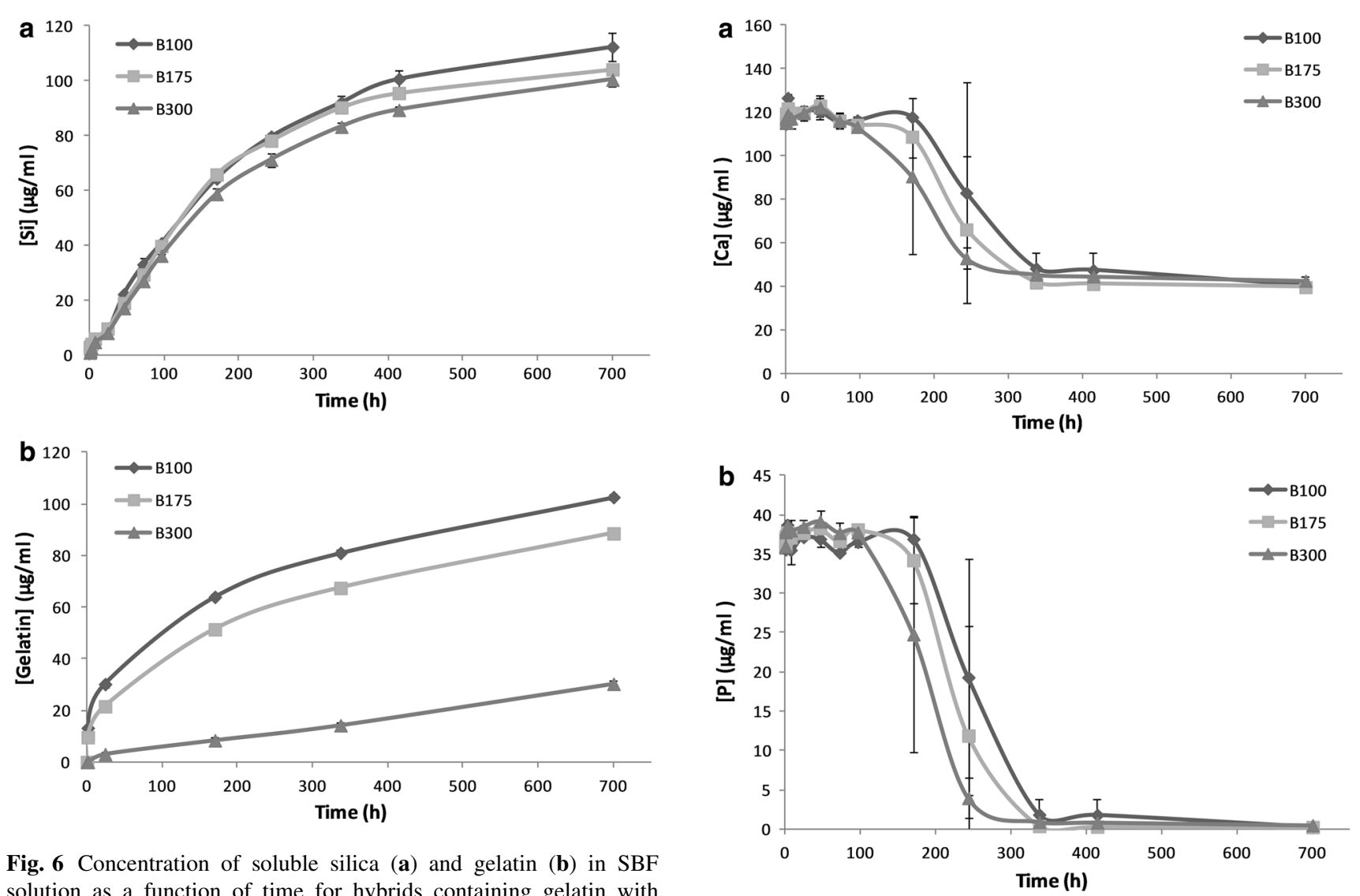

Fig. 6 Concentration of soluble silica (a) and gelatin (b) in SBF solution as a function of time for hybrids containing gelatin with molecular weight as indicated

rather than a change in the degree of condensation of the silica network.

The influence of gelatin molecular weight on hybrid scaffold dissolution in simulated body fluid was investigated in order to establish the role of the organic phase on dissolution. ICP revealed the rate of dissolution of the silica component of the hybrid compared to that of the gelatin phase (measured using a BCA assay, Fig. 6). The release rates of soluble silica across the molecular weight range did not vary by a large extent although there was a small increase in release rate as gelatin molecular weight decreased (Fig. 6a). A similar trend was observed for the release of gelatin (Fig. 6b), however, the range of release rates was considerably greater. After 4 weeks the low molecular weight hybrid released $103 \mu \mathrm{g} \mathrm{ml}^{-1}$ of gelatin compared to $30 \mu \mathrm{g} \mathrm{ml}^{-1}$ for the hybrid containing the high molecular weight gelatin. The result shows that there is minimal change to the release rate of the inorganic phase and therefore the mechanism for dissolution was primarily through hydrolysis of the $\mathrm{Si}-\mathrm{O}-\mathrm{Si}$ bonds between GPTMS and silica. The chains of the organic phase are effectively anchored into the inorganic amorphous network (via GPTMS) and as the shorter polymer chains have fewer of these inorganic 'anchors' per chain (Fig. 1b, c), the release rate of these shorter polymer chains was higher. In

Fig. 7 Concentration of calcium (a) and phosphorus (b) in SBF solution as a function of time for hybrids containing gelatin with molecular weight as indicated

comparison, results from a previous study that showed that increasing covalent coupling through increasing the C-factor (GPTMS concentration) decreased the release rates of both silica and gelatin phases to a similar extent [9]. This is due to the crosslinking effect of GPTMS, which impacts both phases of the hybrid. The chain length $\left(\mathrm{M}_{\mathrm{w}}\right)$ may also directly influence the stability of the gelatin network. If a high proportion of chains are below the entanglement value for gelatin, those smaller chains would be lost from the interpenetrating networks more readily.

Data from ICP (Fig. 7) indicated calcium phosphate precipitation onto the hybrids after 4 days in SBF, through the decrease in calcium and phosphorous content in the SNF over time, with the phosphate content of the SBF approaching zero after $330 \mathrm{~h}$ immersion. This highlights the ability of the hybrids to nucleate a mineral phase throughout the scaffold's surface despite the lack of calcium or phosphate presence within the material. The presence of hydroxyapatite deposits on the scaffold surface was confirmed with XRD and SEM, where this was found to be the predominant mineral phase (Fig. 8). SEM also showed the nature of scaffold degradation, which left a polymeric like 

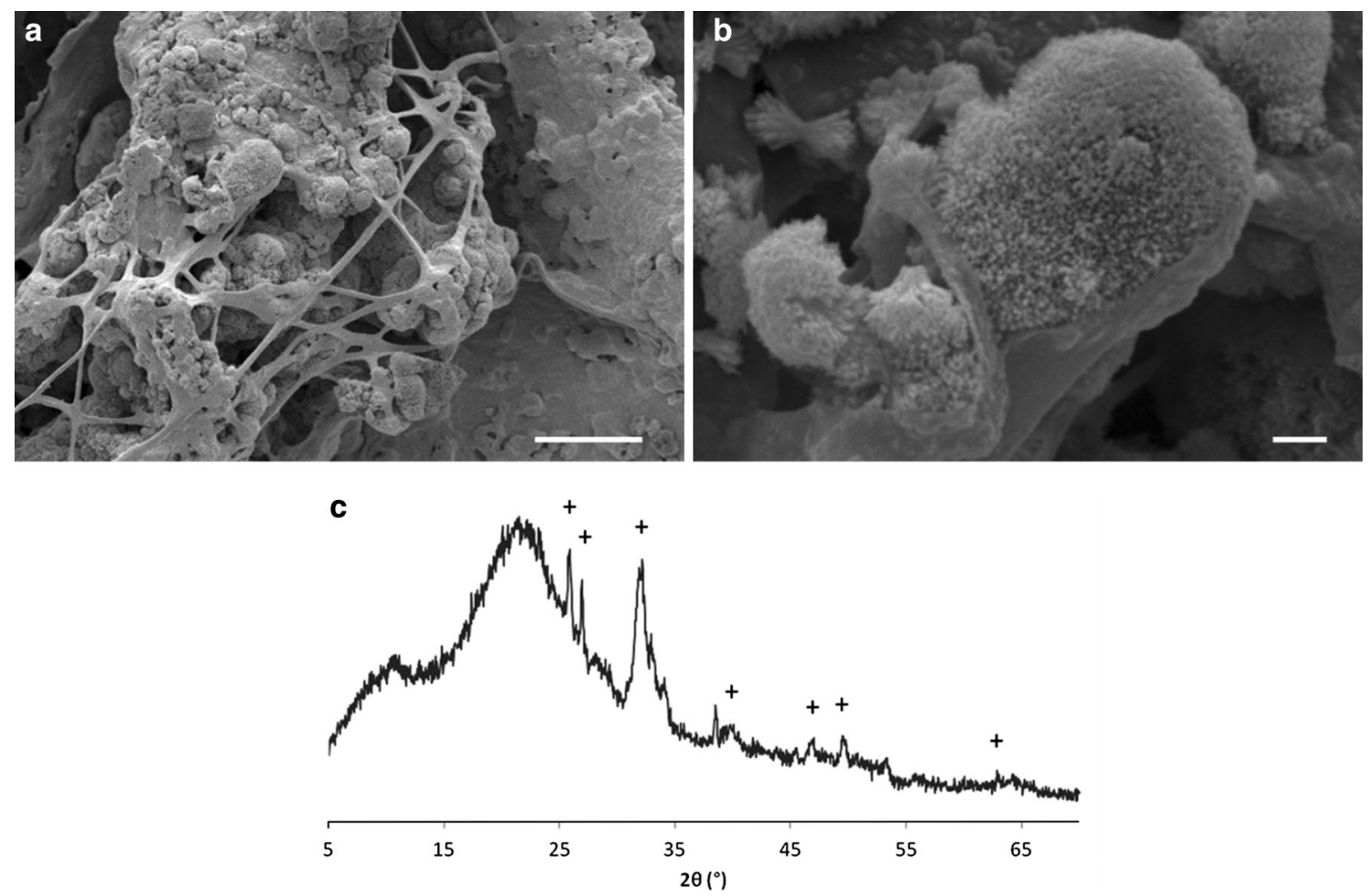

Fig. 8 Hybrid B300 after 20 days immersion in SBF. The crystalline mineral phase is amongst the partially degraded hybrid surface displayed at low (a) and high (b) magnification (scale bar is 10 and
$1 \mu \mathrm{m}$ respectively). XRD revealed the prominent crystal phase was attributed to hydroxyapatite (c). Hydroxyapatite diffraction peaks are marked with a 'plus' symbol

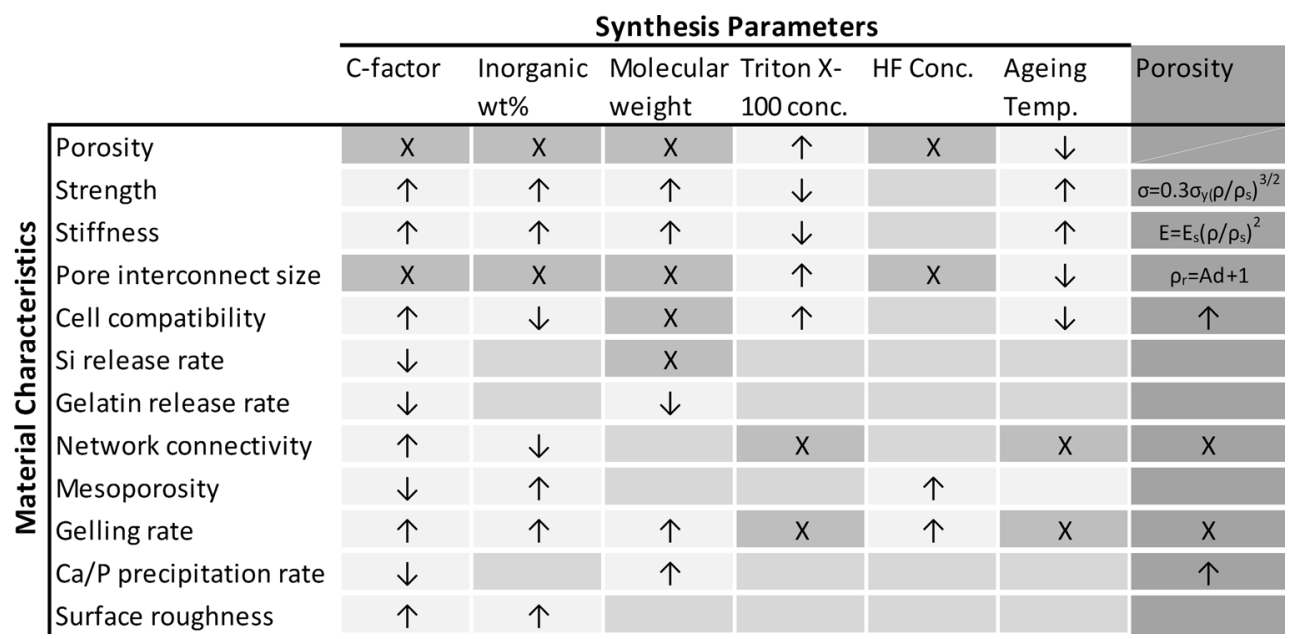

Fig. 9 Matrix of synthesis parameters and corresponding relationship with material characteristics for sol-gel foamed hybrids. Uparrow denotes a positive correlation, downarrow denotes a negative correlation and $X$ denotes no correlation. Empty boxes signify that

matrix in places and provided nucleation sites for clusters of needle like apatite phases. Nucleation on the organic phase was likely to be promoted by the presence of carboxylate groups [40,41] whilst on the inorganic phase silanol groups are well known to initiate apatite nucleation [6]. The data indicates a marginally quicker calcium phosphate deposition there was insufficient information to determine a correlation. Porosity is an important factor in scaffold design. Therefore correlations with porosity were included in the matrix even though it is not a synthesis parameter. Additional data were obtained from [9]

on the samples containing high molecular weight gelatin as the initial precipitation time for the high molecular weight sample was 7 days compared to 10 days for the low molecular weight sample. This was possibly due to the slower dissolution rate of this sample leading to more stable nucleation sites. 


\subsection{A tool for hybrid design}

One of the primary aims of changing the gelatin molecular weight in this study was to achieve a way of controlling certain material characteristics, namely strength and stiffness, without affecting the material's surface chemistry, thereby creating a scaffold that can exhibit different mechanical properties but present the same surface chemistry to cells. This is desirable for either in vitro cell culture studies or in fine tuning a tissue regenerating hybrid scaffold for a specific application in situ. In the design of silica-gelatin hybrid scaffolds for particular applications a key material property may need to be optimised for that application. But, due to the complex nature of hybrid materials, altering this property may or may not influence other key material properties. In order to better understand the influence of hybrid material properties over each other a matrix was generated (Fig. 9), which demonstrates the interdependency of various properties and independence of others. The matrix was compiled based on all data currently available for silica-gelatin hybrid scaffolds. This matrix is an essential tool in optimising scaffolds for specific applications or in experimental design. For instance, if there was a need to investigate the effect of gelatin release rate but not silica release the matrix shows that this is carried out by modifying the molecular weight of gelatin. However, if there was a need to compare results with the combined effect of gelatin and silica release rate then the hybrid's C-factor should be modified. As more data is gathered on silica-gelatin hybrids this matrix can grow to incorporate other important properties.

\section{Conclusion}

A new method to control the material properties of silicagelatin hybrids scaffolds for tissue regeneration has been demonstrated. The influence of gelatin molecular weight over mechanical properties and dissolution rate highlights its importance due to its ability to control key material properties without altering the material chemistry. This enables material properties to be altered while maintaining constant cell-material interaction characteristics with the scaffold.

The processing methods presented in this study enable the generation of highly porous scaffold structures with up to $97.7 \%$ porosity and a modal pore interconnect diameter of $360 \mu \mathrm{m}$. This incredibly open scaffold network is ideal for high cell penetration. Also, as porosity is such a key factor in determining the scaffold mechanical properties, the ability to generate such highly porous scaffolds shows great scope for fine tuning the mechanical properties.
The influence of the gelatin molecular weight over important processing parameters such as gelling rate were also investigated and contributed to the development of a matrix of properties relating to the main synthesis parameters. The matrix can be used as a design tool for silicagelatin hybrids for different tissue engineering applications and will serve as an indispensable tool for designing future hybrid scaffolds.

Acknowledgments The authors would like to acknowledge the EPSRC PhD Plus Scheme and EP/E057091/1, EP/E051669/1 and EP/ I020861/1. JVH and MES thank EPSRC and the University of Warwick for partial funding of the solid state NMR infrastructure at Warwick, and acknowledges additional support for this infrastructure obtained through Birmingham Science City: Innovative Uses for Advanced Materials in the Modern World (West Midlands Centre for Advanced Materials Projects 1 and 2), with support from Advantage West Midlands (AWM) and partial funding by the European Regional Development Fund (ERDF). 3D imaging was carried out at the Manchester X-ray Imaging Facility, which was funded in part by the EPSRC (EP/I02249X/1).

Open Access This article is distributed under the terms of the Creative Commons Attribution License which permits any use, distribution, and reproduction in any medium, provided the original author(s) and the source are credited.

\section{References}

1. Husing N, Schubert U, Misof K, Fratzl P (1998) Formation and structure of porous gel networks from $\mathrm{Si}(\mathrm{OMe})(4)$ in the presence of $\mathrm{A}(\mathrm{CH} 2)(\mathrm{n}) \mathrm{Si}(\mathrm{OR})(3) \quad(\mathrm{A}=$ functional group $)$. Chem Mater 10(10):3024-3032. doi: $10.1021 / \mathrm{cm} 980706 \mathrm{~g}$

2. Kickelbick G (2008) The search of a homogeneously dispersed material - the art of handling the organic polymer/metal oxide interface. J Sol-Gel Sci Technol 46(3):281-290. doi:10.1007/ s10971-008-1731-2

3. Novak BM (1993) Hybrid nanocomposite materials-between inorganic glasses and organic polymers. Adv Mater 5(6):422-433. doi:10.1002/adma.19930050603

4. Poologasundarampillai G, Yu B, Tsigkou O, Valliant EM, Yue S, Lee PD, Hamilton RW, Stevens MM, Kasuga T, Jones JR (2012) Bioactive silica-poly(g-glutamic acid) hybrids for bone regeneration: effect of covalent coupling on dissolution and mechanical properties and fabrication of porous scaffolds. Soft Matter 8(17):4822-4832. doi:10.1039/c2sm00033d

5. Poologasundarampillai G, Ionescu C, Tsigkou O, Murugesan M, Hill RG, Stevens MM, Hanna JV, Smith ME, Jones JR (2010) Synthesis of bioactive class II poly(gamma-glutamic acid)/silica hybrids for bone regeneration. J Mater Chem 20(40):8952-8961. doi:10.1039/c0jm00930j

6. Rhee SH, Lee YK, Lim BS (2004) Evaluation of a novel poly(epsilon-caprolactone)-organosiloxane hybrid material for the potential application as a bioactive and degradable bone substitute. Biomacromolecules 5(4):1575-1579

7. Valliant EM, Jones JR (2011) Softening bioactive glass for bone regeneration: sol-gel hybrid materials. Soft Matter 7(11):5083-5095. doi:10.1039/c0sm01348j

8. Jones JR (2013) Review of bioactive glass: from Hench to hybrids. Acta Biomater 9(1):4457-4486. doi:10.1016/j.actbio. 2012.08.023 
9. Mahony O, Tsigkou O, Ionescu C, Minelli C, Hanly R, Ling L, Smith ME, Stevens MM, Jones JR (2010) Silica-gelatin hybrids with tailorable degradation and mechanical properties for tissue regeneration. Adv Funct Mater 20(22):3835-3845. doi:10.1002/ adfm. 201000838

10. Nicole L, Boissiere C, Grosso D, Quach A, Sanchez C (2005) Mesostructured hybrid organic-inorganic thin films. J Mater Chem 15(35-36):3598-3627. doi:10.1039/b506072a

11. Chen S, Osaka A, Ikoma T, Morita H, Li J, Takeguchi M, Hanagata N (2011) Fabrication, microstructure, and BMP-2 delivery of novel biodegradable and biocompatible silicate-collagen hybrid fibril sheets. J Mater Chem 21(29):10942-10948. doi:10. 1039/c1jm10829h

12. Fatimi A, Tassin JF, Quillard S, Axelos MAV, Weiss P (2008) The rheological properties of silated hydroxypropylmethylcellulose tissue engineering matrices. Biomaterials 29(5):533-543. doi:10. 1016/j.biomaterials.2007.10.032

13. Peng FB, Lu LY, Sun HL, Wang YQ, Liu JQ, Jiang ZY (2005) Hybrid organic anorganic membrane: solving the tradeoff between permeability and selectivity. Chem Mater 17(26):6790-6796. doi: $10.1021 / \mathrm{cm} 051890 \mathrm{q}$

14. Liu YL, Su YH, Lai JY (2004) In situ crosslinking of chitosan and formation of chitosan-silica hybrid membranes with using gamma-glycidoxypropyltrimethoxysilane as a crosslinking agent. Polymer 45(20):6831-6837. doi:10.1016/j.polymer.2004.08.006

15. Green WH, Le KP, Grey J, Au TT, Sailor MJ (1997) White phosphors from a silicate-carboxylate sol-gel precursor that lack metal activator ions. Science 276(5320):1826-1828. doi:10.1126/ science.276 5320.1826

16. Lenza RFS, Vasconcelos WL, Jones JR, Hench LL (2002) Surface-modified 3D scaffolds for tissue engineering. J Mater Sci Mater Med 13(9):837-842

17. Maeda H, Kasuga T, Hench LL (2006) Preparation of poly(Llactic acid)-polysiloxane-calcium carbonate hybrid membranes for guided bone regeneration. Biomaterials 27(8):1216-1222. doi:10.1016/j.biomaterials.2005.08.010

18. Rhee SH, Choi JY, Kim HM (2002) Preparation of a bioactive and degradable poly(epsilon-caprolactone)/silica hybrid through a sol-gel method. Biomaterials 23(24):4915-4921. doi:10.1016/ s0142-9612(02)00251-x

19. Yoo JJ, Rhee SH, Lee JI, Kim HJ (2009) Evaluations of bioactive poly(epsilon-caprolactone)-organosiloxane nano-hybrid as a material for bone repair. Tissue Eng Regen Med 6(4-11):445-452

20. Ren L, Tsuru K, Hayakawa S, Osaka A (2002) Novel approach to fabricate porous gelatin-siloxane hybrids for bone tissue engineering. Biomaterials 23(24):4765-4773

21. Gabrielli L, Russo L, Poveda A, Jones JR, Nicotra F, JimenezBarbero J, Cipolla L (2013) Epoxide opening versus silica condensation during sol-gel hybrid biomaterial synthesis. Chem Eur J 19(24):7856-7864. doi:10.1002/chem.201204326

22. Heino J (2007) The collagen family members as cell adhesion proteins. BioEssays 29(10):1001-1010. doi:10.1002/bies.20636

23. Radin S, El-Bassyouni G, Vresilovic EJ, Schepers E, Ducheyne P (2005) In vivo tissue response to resorbable silica xerogels as controlled-release materials. Biomaterials 26(9):1043-1052. doi:10.1016/j.biomaterials.2004.04.004

24. Midha S, van den Bergh W, Kim TB, Lee PD, Jones JR, Mitchell CA (2013) Bioactive glass foam scaffolds are remodelled by osteoclasts and support the formation of mineralized matrix and vascular networks in vitro. Adv Healthc Mater 2(3):490-499. doi:10.1002/adhm.201200140

25. Jones JR, Tsigkou O, Coates EE, Stevens MM, Polak JM, Hench LL (2007) Extracellular matrix formation and mineralization of on a phosphate-free porous bioactive glass scaffold using primary human osteoblast (HOB) cells. Biomaterials 28:1653-1663

26. Yue S (2011) Non-Destructive quantification of tissue scaffolds and augmentation implants using X-ray microtomography. $\mathrm{PhD}$ thesis, Imperial College London, London

27. Yue S, Lee PD, Poologasundarampillai G, Jones JR (2011) Evaluation of 3-D bioactive glass scaffolds dissolution in a perfusion flow system with X-ray microtomography. Acta Biomater 7(6):2637-2643. doi:10.1016/j.actbio.2011.02.009

28. Kokubo T, Ito S, Huang ZT, Hayashi T, Sakka S, Kitsugi T, Yamamuro T (1990) Ca, P-rich layer formed on high-strength bioactive glass-ceramic A-W. J Biomed Mater Res 24(3):331-343

29. Gibson LJ, Ashby MF (1988) Cellular solids structure and properties. Pergamon Press, Oxford

30. MacKenzie KJD, Smith ME (2002) Multinuclear solid state NMR of inorganic materials. Pergamon Press, Oxford

31. Sepulveda P, Jones JR, Hench LL (2002) Bioactive sol-gel foams for tissue repair. J Biomed Mater Res 59(2):340-348

32. Jones JR, Ehrenfried LM, Hench LL (2006) Optimising bioactive glass scaffolds for bone tissue engineering. Biomaterials 27:964-973

33. Jones JR, Hench LL (2003) Effect of surfactant concentration and composition on the structure and properties of sol-gel-derived bioactive glass foam scaffolds for tissue engineering. J Mater Sci 38(18):3783-3790

34. Jones JR, Hench LL (2004) Factors affecting the structure and properties of bioactive foam scaffolds for tissue engineering. J Biomed Mater Res B 68B(1):36-44

35. Lin S, Ionescu C, Pike KJ, Smith ME, Jones JR (2009) Nanostructure evolution and calcium distribution in sol-gel derived bioactive glass. J Mater Chem 19(9):1276-1282

36. Hulbert SF, Morrison SJ, Klawitte JJ (1972) Tissue reaction to three ceramics of porous and non-porous structures. J Biomed Mater Res 6(5):347-374

37. Holy CE, Shoichet MS, Davies JE (2000) Engineering threedimensional bone tissue in vitro using biodegradable scaffolds: investigating initial cell-seeding density and culture period. J Biomed Mater Res 51(3):376-382

38. Carter DR, Hayes WC (1976) Bone compressive strength-influence of density and strain rate. Science 194(4270):1174-1176. doi:10.1126/science. 996549

39. Hench LL, West JK (1990) The sol-gel process. Chem Rev 90(1):33-72

40. Hunter GK, Goldberg HA (1994) Modulation of crystal-formation by bone phosphoproteins-role of glutamic acid-rich sequences in the nucleation of hydroxyapatite by bone sialoprotein. Biochem J 302:175-179

41. Ohtsuki C, Kamitakahara M, Miyazaki T (2007) Coating bonelike apatite onto organic substrates using solutions mimicking body fluid. J Tissue Eng Regen Med 1(1):33-38. doi:10.1002/ term.3 